\title{
Pankreatitis beim Hund
}

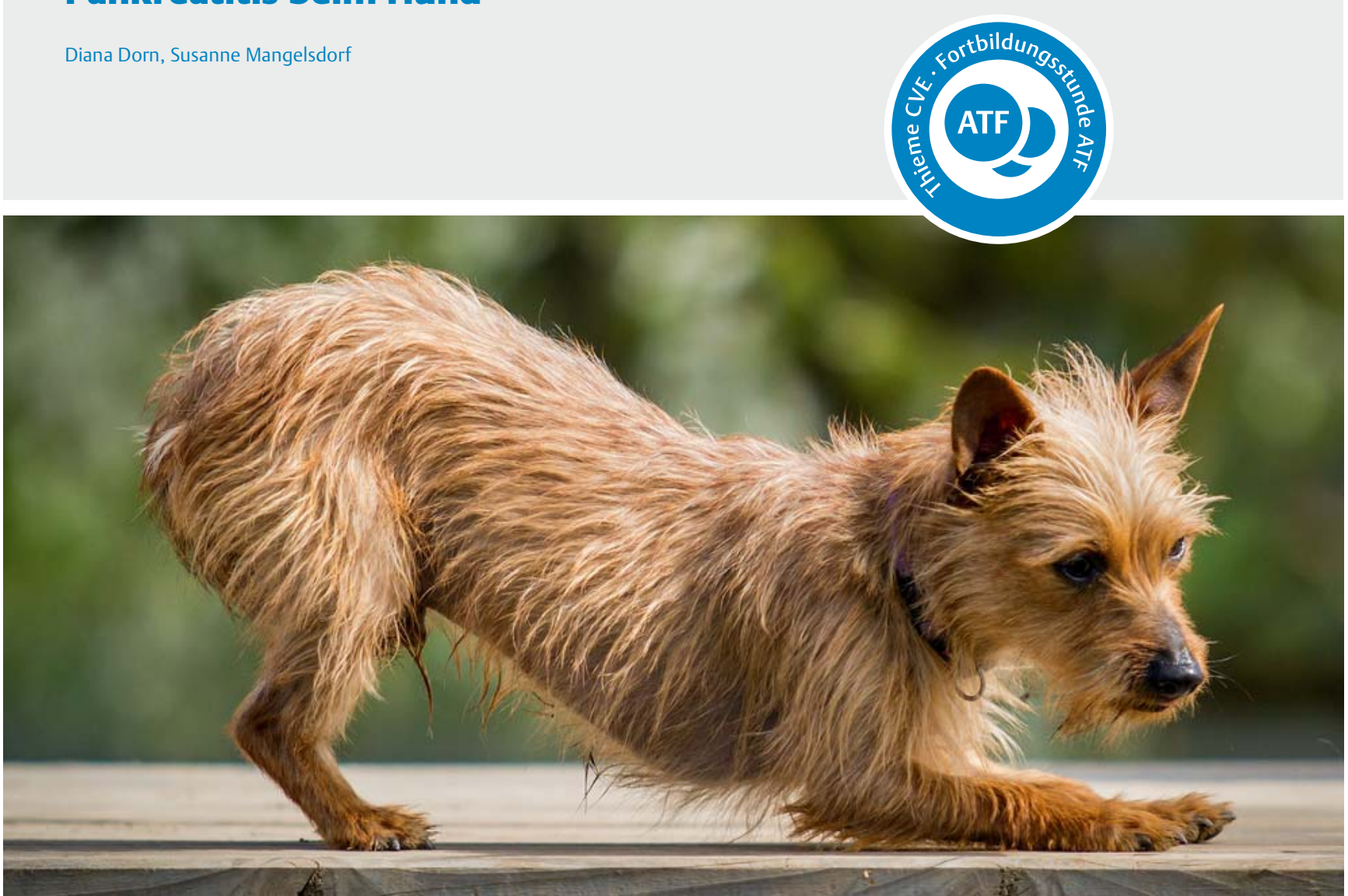

(c) stock.adobe.com - hemlep

Die Pankreatitis ist die häufigste Erkrankung des exokrinen Pankreas des Hundes. Die Diagnose stellt aufgrund der unterschiedlichen klinischen Präsentation eine Herausforderung dar. In diesem Artikel wird detailliert erläutert, welche diagnostischen Untersuchungen sinnvoll sind und welche therapeutischen Möglichkeiten zur Verfügung stehen.

\section{Einleitung}

Eine Pankreatitis beschreibt die entzündliche Infiltration des exokrinen Pankreas. Es werden die akute und die chronische Form unterschieden [1,2]. Die akute Pankreatitis wird definiert als eine potenziell reversible Entzündung mit histologisch nachweisbarer Ödembildung, Infiltration von Neutrophilen und Nekrose. Sie kann lokal begrenzt ablaufen oder zu einer systemischen Entzündungsreaktion (Systemic Inflammatory Response Syndrome, SIRS) mit Multiorganversagen führen.
Die chronische Verlaufsform ist histologisch durch eine lymphozytäre oder lymphoplasmazelluläre Infiltration und irreversible Schäden wie Fibrose gekennzeichnet [3].

Die genaue Ätiologie beider Verlaufsformen ist noch nicht eindeutig geklärt. Viele unterschiedliche Risikofaktoren, die die Entstehung begünstigen, sind jedoch bekannt.

Zu den wichtigsten gehören:

- Rasse, z. B. Zwergschnauzer, Sheltie, Cavalier King Charles, Cocker Spaniel

- Hypertriglyzeridämie 
- Tab. 1 Häufige Symptome akuter und chronischer Pankreatitis beim Hund.

\begin{tabular}{l|l|}
\hline akute Pankreatitis & chronische Pankreatitis \\
\hline - Anorexie & - subklinischer Verlauf ohne \\
\hline - Vomitus & deutliche Symptome \\
\hline - Schwäche & - intermittierende Anorexie \\
\hline - Diarrhoe & - Schwäche \\
\hline - schmerzhaftes & - schmerzhaftes Abdomen \\
& Abdomen \\
- Ikterus & - Gewichtsverlust \\
- Fieber & \\
- Dehydratation &
\end{tabular}

- Adipositas

- ungeeignete Futtermittel (Abfall, Tischreste)

- Infektionen, z. B. Babesiose

- Medikamente, z. B. Phenobarbital, Kaliumbromid

- Intoxikationen, z.B. Zink, Organophosphate

- Endokrinopathien, z. B. Hyperadrenokortizismus, Hypothyreose, Diabetes mellitus

- direkte Traumatisierung des Pankreas (abdominale Traumata, Operationen)

Auch Hypoxie und Hypoperfusion können zu einer Schädigung des Pankreas führen.

Die chronische Pankreatitis kann als Komplikation einer akuten Pankreatitis auftreten $[3,4]$. Sie wird aber auch als Folge einer chronischen, immunvermittelten Entzündung, wie sie beim Englischen Cocker Spaniel beschrieben ist, diskutiert $[5,6]$.

\section{Klinik}

Die klinischen Symptome an Pankreatitis erkrankter Hunde variieren deutlich je nach Verlaufsform (

Hunde mit einer akuten Pankreatitis zeigen vor allem abdominale Schmerzen, Vomitus und Anorexie. Auch Diarrhoe kommt häufig vor und sollte als wichtige Differenzialdiagnose für eine Pankreatitis gesehen werden.

Die Befunde der klinischen Untersuchung sind abhängig vom Schweregrad der Erkrankung sowie dem Ausmaß der Dehydratation. Ein starker Flüssigkeitsverlust durch Erbrechen und/oder Durchfall kann zu einem hypovolämischen Schock führen. Patienten mit fulminanter Pankreatitis können zudem mit Symptomen einer SIRS oder disseminierten intravasalen Gerinnung (DIC) vorgestellt werden. Eine SIRS ist charakterisiert durch mikrovaskuläre Thrombosierung sowie erhöhte Permeabilität der kapillären Gefäße, die durch eine Vielzahl inflammatorischer Zytokine verursacht wird. Typische Symptome einer SIRS lassen sich in 2 Phasen gliedern.
Die initiale hyperdynamische Phase ist gekennzeichnet durch:

- Tachykardie (>160/min)

- Hyperthermie $\left(>39,7^{\circ} \mathrm{C}\right)$

- Polypnoe (>40/min)

- hyperämische Schleimhäute durch eine periphere Vasodilatation

Die 2. Phase, die der Hypoperfusion, kennzeichnet sich durch:

- Hypothermie $\left(<37,7^{\circ} \mathrm{C}\right)$

- Tachykardie

- blasse Schleimhäute mit verlängerter kapillärer Füllungszeit

- schlechte Pulsqualität

- reduzierte Bewusstseinslage

Bei einer DIC kommt es je nach Stadium zu Mikrothrombosierungen oder spontanen Blutungen. Eine SIRS oder DIC im Verlauf einer fulminanten Pankreatitis resultieren häufig in einem Multiorganversagen [4, 7, 8].

Hunde mit einer chronischen Verlaufsform können lange subklinisch bleiben oder nur milde, unspezifische Symptome aufweisen. Bleibt die chronische Pankreatitis unentdeckt, kann die einsetzende Fibrosierung zum Verlust des Pankreasgewebes und somit im Endstadium zu einer exokrinen Pankreasinsuffizienz sowie, in seltenen Fällen, einem Diabetes mellitus führen [3].

\section{Diagnostik}

\section{Allgemeine Untersuchung}

Da die Ursachen bzw. die Risikofaktoren sowie die Symptome einer Pankreatitis vielfältig sind, gehört zur Diagnostik eine ausführliche Anamnese sowie eine vollständige klinische Untersuchung. Dadurch ergeben sich gegebenenfalls bereits erste Hinweise auf eine bestehende Grunderkrankung oder eine mögliche Aufnahme von inadäquatem Futter oder toxischen Substanzen.

Betroffene Tiere zeigen unter Umständen Haltungsveränderungen. Viele Hunde mit starken abdominalen Schmerzen nehmen eine Gebetshaltung ein oder zeigen einen aufgekrümmten Rücken sowie Schmerzäußerungen bei abdominaler Palpation. Ein Ikterus kann ein Hinweis auf eine Pankreatitis sein. Patienten mit einem Flüssigkeitsdefizit zeigen pappige Schleimhäute mit einer verlängerten kapillären Füllungszeit. Auch Fieber kann im Rahmen der Pankreatitis auftreten.

\section{Allgemeine Laboruntersuchung}

Bei allen Patienten mit dem Verdacht auf eine Pankreatitis sollten ein Blutbild, eine Blutchemie sowie Elektrolyte und eine Urinuntersuchung durchgeführt werden. Diese 
Tests sind nicht primär zur Diagnostik einer Pankreatitis geeignet.

Sie erlauben dem Tierarzt jedoch eine bessere Einschätzung von:

- Hydratationsstatus

- Organfunktion

- vorliegenden Grunderkrankungen

- Differenzialdiagnosen

- Krankheitsstadium

- möglichem Verlauf der Pankreatitis

Hunde mit einer subklinisch verlaufenden Pankreatitis zeigen oft nur milde oder unspezifische Veränderungen. Patienten mit schwerem Verlauf einer akuten Pankreatitis mit systemischen Komplikationen zeigen hingegen deutliche Veränderungen sowohl im Blutbild als auch in der Blutchemie. Diese Veränderungen sind jedoch nicht pathognomonisch für eine Pankreatitis, sondern treten meist sekundär auf [14].

Einer Studie zufolge, die 70 Hunde mit einer schweren akuten Pankreatitis untersuchte, waren die häufigsten hämatologischen Veränderungen Thrombozytopenie, Leukozytose sowie eine Neutrophilie mit Kernlinksverschiebung [9]. Eine deutliche Leukozytose mit Linksverschiebung sowie eine Leukopenie in Verbindung mit entsprechenden klinischen Symptomen sind Hinweise auf eine mögliche Sepsis oder SIRS. Im Chemieprofil treten häufig Leberenzymerhöhungen und Bilirubinämie auf.

\section{PRAXISTIPP}

Eine Bilirubinämie in Kombination mit einem cholestatischen Leberenzymprofil sollte immer Anlass zur Abklärung einer Pankreatitis sein, da es aufgrund der anatomischen Nähe zur Mündung des Ductus choledochus communis zu einer extrahepatischen Gallengangsobstruktion kommen kann.

Eine Erhöhung der Kreatinin- und/oder Harnstoffkonzentrationen spiegelt meist einen Flüssigkeitsverlust durch Vomitus und Diarrhoe wider. In schweren Fällen kann dies jedoch auch der Hinweis auf ein akutes Nierenversagen sein [5].

\section{Merke}

Zur Unterscheidung einer prärenalen und renalen Azotämie ist eine Urinuntersuchung notwendig.

Andere mögliche Auffälligkeiten in der Blutchemie sind:

- Hypoalbuminämie

- Hypertriglyzeridämie

- Hypercholesterolämie

- Hyper- oder Hypoglykämie
Elektrolytveränderungen werden ebenfalls häufig gesehen, wobei eine Hypokaliämie sowie geringgradige Hyponatriämie am häufigsten beobachtet werden [5]. Diese Abweichungen sind meist Folge von Erbrechen, Durchfall und verminderter Futteraufnahme.

\section{Spezielle Laboruntersuchung}

Pankreatische Lipase (PL)

Die pankreatische Lipase ist eine spezifische Lipase, die ausschließlich vom Pankreas gebildet wird. Sie wird bei entzündlichen Prozessen in kleinen Mengen in den systemischen Blutkreislauf abgegeben.

Die PL kann mittels Radioimmunassay (cPLI) und mittels ELISA (CPL) gemessen werden. Durch den Einsatz von Antikörpern ist die Messung speziesspezifisch. Die Messung der caninen Pankreaslipase cPL (cPLI, Spec cPL) ist eine Laboruntersuchung mit guter Sensitivität und Spezifität zur Diagnose einer Pankreatitis. Dennoch kann es auch mit dieser Methode falsch positive und falsch negative Werte geben.

Die Sensitivität des Tests hängt stark vom Schweregrad der Erkrankung ab und wird in der Literatur zwischen $21 \%$ und $71 \%$ angegeben [4]. Sie erscheint höher bei Patienten mit einer schweren akuten Pankreatitis. Dies liegt wahrscheinlich darin begründet, dass die Enzyme aus einem stark entzündlich veränderten Gewebe stärker austreten. Die Spezifität liegt bei bis zu 86\% [4|. Bei Patienten mit einer akuten moderaten bis schweren Pankreatitis ist die Bestimmung der caninen Pankreaslipase damit ein sinnvoller Test zur Diagnostik.

\section{PRAXISTIPP}

Die Messung der PLI sollte nach 8-12 Stunden Nahrungskarenz erfolgen.

Zur semiquantitativen Bestimmung der $\mathrm{CPL}$ existiert ein Schnelltest (SNAP cPL, IDEXX). Dieser Test basiert auf der gleichen Methode wie die quantitative Bestimmung. Der Test beinhaltet einen Referenzpunkt, der dem oberen Wert des Referenzintervalls der CPL des Hundes $(200 \mu \mathrm{g} /$ L) entspricht und ist vor allem zum Ausschluss einer Pankreatitis gedacht.

\section{Merke}

Positive Ergebnisse des Schnelltests sollten durch die Bestimmung der CPL bestätigt werden $[10,26]$.

Trypsin-Like Immunoreaktivität (TLI)

Die TLI reflektiert die Funktion des exokrinen Pankreas. Die Sensitivität der Serum-TLI-Konzentration für die akute canine Pankreatitis ist im Vergleich zur cPLI und zur Sonografie deutlich geringer und liegt zwischen 36-47\% $[5,13]$. Dies liegt hauptsächlich darin begründet, dass 
die TLI zu Beginn einer akuten Pankreatitis zwar ansteigt, im Verlauf jedoch relativ zügig wieder abfällt. Die Messung der TLI-Konzentration bleibt vor allem der Test der Wahl für eine exokrine Pankreasinsuffizienz.

\section{Serum-Amylase-Aktivität}

Das Enzym Amylase katalysiert die Hydrolyse von komplexen Kohlenhydraten und wird, neben dem Pankreas, von vielen verschiedenen Zelltypen synthetisiert. Ein deutlicher Anstieg kann hinweisend für eine Pankreatitis sein, jedoch können auch andere gastrointestinale Erkrankungen und Lebererkrankungen eine Erhöhung der Amylase-Aktivität zur Folge haben. Auf der anderen Seite zeigen viele Hunde mit akuter Pankreatitis keinen Anstieg der Amylase [10]. Dieser Test besitzt also eine geringe Sensitivität und Spezifität und wird daher zur Diagnostik einer Pankreatitis nicht mehr empfohlen [11].

\section{Serum-Lipase-Aktivität}

Lipasen hydrolysieren Lipide. Im Körper kommen unterschiedliche Lipasen vor, z.B. gastrische Lipase, pankreatische Lipase, hepatische Lipase und Hormon-sensitive Lipase. Bisher galt die Bestimmung der Serum-LipaseAktivität ähnlich der Amylase weder als sensitiv noch als spezifisch für eine Pankreatitis. Die Aussagekraft der Untersuchung der Serum-Lipase-Aktivität ist auch von dem verwendeten Substrat im Assay zur Untersuchung der Enzym-Aktivität abhängig.

Seit 2001 gibt es ein Assay zur Bestimmung der LipaseAktivität, in dem das Substrat 1,2-o-Dilauryl-rac-glycero-3-glutarsäure-(6-methyl-resorufin)-Ester (DGGR) verwendet wird. Die Enzym-Substrat-Interaktionen in diesem Assay sind selektiver für die pankreatische Lipase [33]. Sensitivität und Spezifität der DGGR-Lipase liegen bei 73,3\% bzw. 66,6\%. Damit ist die DGGR-Lipase ähnlich aussagekräftig wie die Spec cPL $[33,34]$.

\section{Serum-pankreatische Elastase-1 (cPE-1)}

Die cPE-1 wird in den Azinuszellen des Pankreas gebildet und bei einer aktiven entzündlichen Veränderung des Pankreas in das Blut abgegeben. Der Nachweis im Serum erfolgt mittels eines ELISA und ist somit speziesspezifisch. In einer Studie mit 61 Hunden erreichte diese Untersuchung eine Sensitivität von 61,4\% und eine Spezifität von $92 \%$ [35].

Diese Untersuchung der Serum-cPE-1 wird jedoch von vielen Laboren nicht standardmäßig angeboten.

\section{Bildgebende Verfahren}

\section{Röntgen}

Das Röntgen ist eine schnelle Diagnostik, die wichtig ist, um Differenzialdiagnosen eines akuten Abdomens abzuklären. In der abdominalen Röntgenaufnahme können sich bei einer akuten Pankreatitis Veränderungen wie Detailverlust im kranialen Abdomen ( $\bullet$ Abb. 1), die Ver-

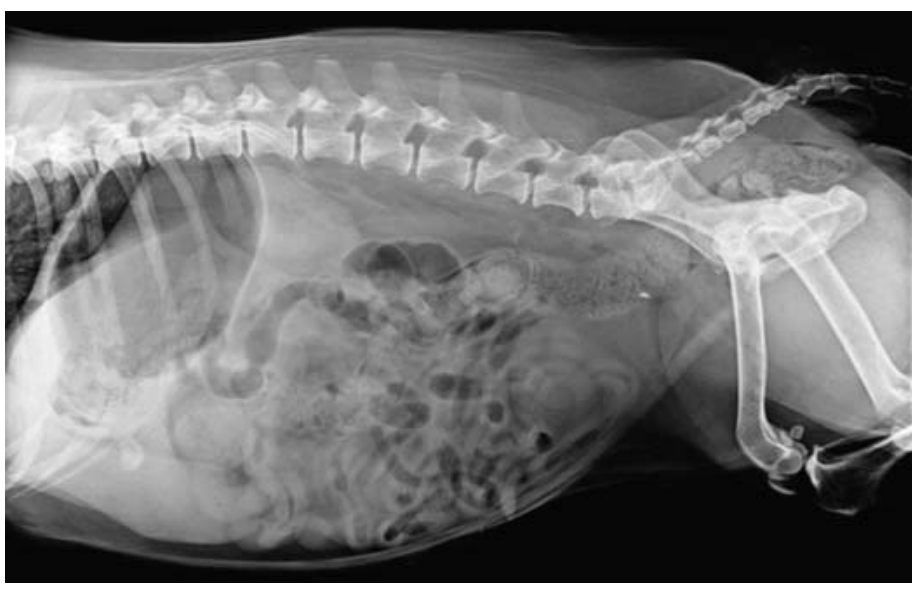

- Abb. 1 Abdomenröntgen im laterolateralen Strahlengang. Neben einer Hepatomegalie zeigen sich auch ein Detailverlust im kranialen Abdomen und luftgefüllte Darmschlingen. @ K. Schmerbach/R. Höpfner

lagerung des Duodenums nach dorsal oder lateral sowie gasgefüllte, dem Pankreas angelagerte Darmschlingen zeigen $[4,5,8]$. Diese Veränderungen sind jedoch nicht spezifisch und reichen für die Diagnose einer Pankreatitis nicht aus.

\section{Ultraschall}

Die Ultraschalluntersuchung ist eine wichtige, nicht invasive Untersuchungsmethode zur Diagnostik der Pankreatitis des Hundes. Die Sensitivität dieser Untersuchung ist stark von der Erfahrung des Untersuchenden und der technischen Ausstattung abhängig [4, 9, 16]. Zur Beurteilung des Pankreas werden Hochfrequenzsonden $(>7,5 \mathrm{MHz}$ ) empfohlen.

Der Untersuchungsgang findet meist in Rückenlage statt. Die anatomischen Orientierungspunkte sind die rechte Niere, das Duodenum descendens und die V. pancreaticoduodenalis. Der Schallkopf wird rechts in Längsachse hinter der letzten Rippe zur Darstellung der rechten Niere platziert und dann nach medial in Richtung des Duodenums descendens geschwenkt. Alternativ kann der Schallkopf unterhalb des Xyphoids angesetzt und in Richtung des Pylorus nach rechts bewegt werden, um das Duodenum descendens und schließlich den Lobus dexter des Pankreas darzustellen. Der Lobus pancreatis sinister ist beim Hund aufgrund der Gasartefakte des Colon transversum häufig nicht gut darstellbar.

Im Normalzustand zeigt sich das Pankreas oft isoechogen zum umliegenden Fettgewebe und lässt sich dann häufig nicht gut abgrenzen ( $\bullet$ Abb. 2). Das Parenchym wirkt homogen [15].

Bei einer akuten Pankreatitis zeigt sich das Pankreas häufig verbreitert, hypoechogen mit unregelmäßigen Grenzen. Das umliegende Fettgewebe ist hyperechogen und 

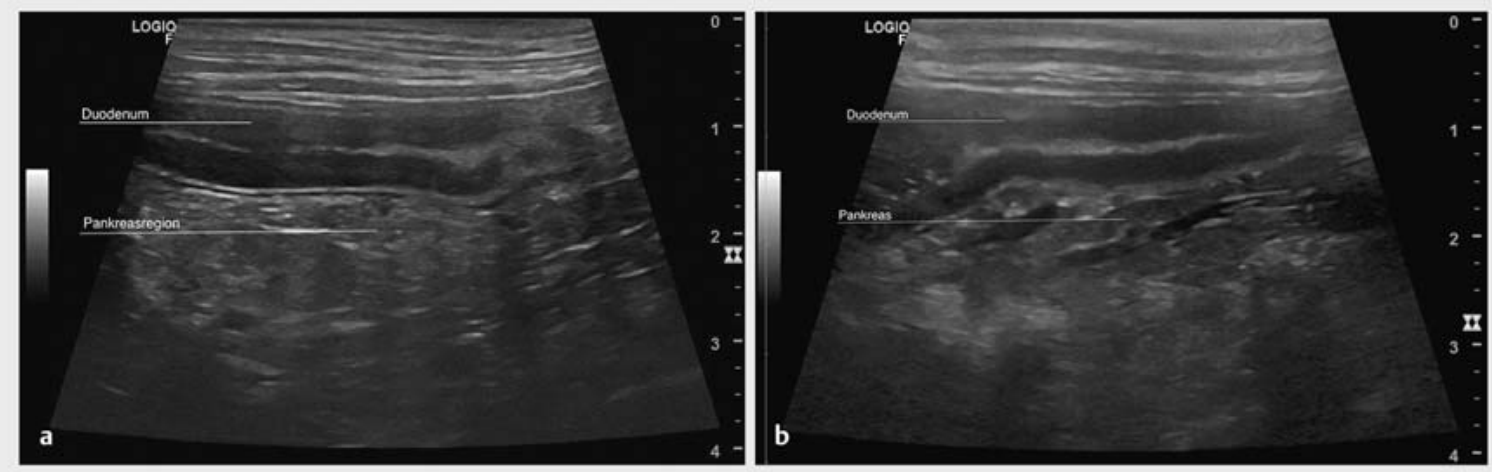

- Abb. 2 Sonografische Darstellung des Pankreas: Physiologisches Pankreas (Lobus pancreatis dexter), das sich nur wenig vom umliegenden Gewebe abgrenzt. @ K. Schmerbach/R. Höpfner

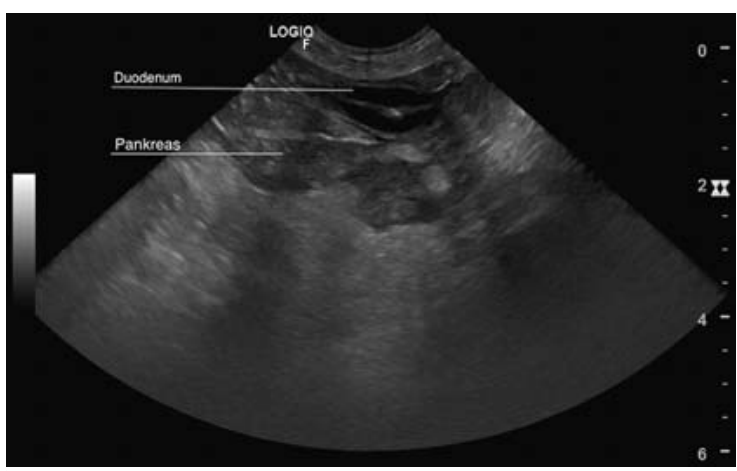

Abb. 3 Sonografische Darstellung einer akuten Pankreatitis. Das Pankreas (Lobus pancreatis dexter) erscheint hypoechogen und verbreitert. Das umliegende Gewebe zeigt sich deutlich hyperechogen. () K. Schmerbach/ R. Höpfner

kann Flüssigkeitseinschlüsse aufweisen ( $\bullet$ Abb. 3). Das anliegende Duodenum descendens kann sich mit einer verdickten Wand, verwaschener Wandschichtung, starr und flüssigkeitsgefüllt oder einem korrugierten (deutlich gewundenen) Verlauf darstellen. Es kann fokal freie Flüssigkeit vorhanden sein. Eine Pankreatitis ist eine häufige Ursache für eine extrahepatische biliäre Obstruktion [4, $5,15]$.

Bei einer chronischen Pankreatitis spielt die Ultraschalluntersuchung eine eher untergeordnete Rolle. Oftmals zeigen sich keine Veränderungen. In einigen Fällen weist das Pankreas eine unregelmäßige Echogenität auf oder erscheint fokal hyperechogen [15].

\section{Computertomografie}

In der Humanmedizin gilt die kontrastmittelgestützte Computertomografie als eine sehr wichtige Untersuchungsmethode zur Erkennung akuter und chronischer Pankreatitiden mit hoher Sensitivität und Spezifität [1721].

\section{CT IN DER TIERMEDIZIN}

In einer Pilotstudie mit 10 Hunden mit bestätigter Pankreatitis wurde eine computertomografische Angiografie durchgeführt. In dieser Studie war die kontrastmittelgestützte Computertomografie dem UItraschall überlegen, da das gesamte Pankreas und die Umgebung eingesehen werden konnten und die Art der Kontrastmittelanreicherung auch einen Hinweis auf die Stärke der Entzündung gab [22]. In der abdominalen Sonografie sind oft nicht alle Bereiche des Pankreas einsehbar durch Abdominalschmerz, einen stark flüssigkeitsgefüllten Magen und/oder Gasartefakte. Aufgrund der kleinen Fallzahlen und der fehlenden Vergleichswerte gesunder Hunde ist diese Methode noch nicht der diagnostische Standard für eine Pankreatitis. Sie ist außerdem aufwendig und kostenintensiv und stellt zudem aufgrund einer notwendigen Sedation oder Narkose ein zusätzliches Risiko dar.

\section{Zytologische und histopathologische Untersuchung}

Zytologische Untersuchung

Die Feinnadelaspiration (FNA) des Pankreas ist eine minimalinvasive, risikoarme diagnostische Maßnahme. So zeigten sich in einer Studie an gesunden Hunden keine Veränderungen am Gewebe oder der cPLI nach einer Punktion und Aspiration [23].

\section{PRAXISTIPP}

Die zytologische Untersuchung kann vor allem bei der Unterscheidung von neoplastischen und nicht neoplastischen Veränderungen sehr hilfreich sein [23]. 
Bei Vorliegen einer akuten Pankreatitis zeigt sich neben einer Infiltration von Neutrophilen eine insgesamt erhöhte Zellularität und Zelldebris aufgrund von Nekrose [5]. Das erschwert die Interpretation des Punktats, und eine fehlende neutrophile Infiltration schließt das Vorliegen einer Pankreatitis nicht aus [10].

Bei Vorliegen einer chronischen Pankreatitis besteht meist eine geringere Zellularität, in geringer Anzahl kommen Neutrophile und Lymphozyten vor [5].

Die sichere Diagnose einer akuten und chronischen Pankreatitis ist zytologisch nicht möglich.

\section{Histopathologische Untersuchung}

Die histopathologische Untersuchung gilt seit langer Zeit als Goldstandard für die definitive Diagnose der Pankreatitis und die Unterscheidung zwischen einer akuten und chronischen Verlaufsform. Es ist bisher jedoch noch nicht geklärt, wie histopathologische Veränderungen (Entzündungen, Fibrose) an klinisch und labordiagnostisch unauffälligen Hunden interpretiert werden sollten.

Letztendlich ist die chirurgische Probennahme am Pankreas eine invasive Untersuchungsmethode, die eine Narkose erfordert. Patienten mit einer schweren akuten Pankreatitis haben ein erhöhtes Narkoserisiko. Außerdem kann die Narkose selbst einen Risikofaktor zur Verschlechterung der Pankreatitis darstellen $[5,10]$. Eine Probennahme bei einer akuten Pankreatitis ist daher nur in Ausnahmefällen gerechtfertigt, vor allem dann, wenn eine operative Versorgung aufgrund von sekundär auftretenden Komplikationen notwendig erscheint. Im Gegensatz dazu ist die sichere Diagnose einer chronischen Pankreatitis oftmals nur durch eine histopathologische Untersuchung des Pankreas möglich.

\section{UNTERSUCHUNGEN}

Die sichersten Verfahren zur Diagnose einer akuten Pankreatitis sind neben einer guten Allgemeinuntersuchung und allgemeinen Laboruntersuchung die Bestimmung der DGGR-Lipase oder CPL und eine Ultraschalluntersuchung des Abdomens. Der SNAPTest kann zum Ausschluss einer Pankreatitis verwendet werden. Die Amylase sollte nicht zur Diagnostik einer Pankreatitis genutzt werden.

Die chronische Pankreatitis kann in ihren Symptomen einer chronischen Enteropathie wie der IBD (Inflammatory Bowel Disease) ähneln. Die sicherste Diagnose in diesem Fall ist die histopathologische Beurteilung einer Biopsie des Pankreas.

\section{Therapie}

Gemäß den oben genannten Risikofaktoren sollten zugrundeliegende Erkrankungen behandelt und andere begünstigende Faktoren, z. B. inadäquate Ernährung, Medikamentengabe, abgestellt oder angepasst werden. Die Therapie der Pankreatitis erfolgt vor allem symptomatisch und richtet sich nach dem in der Allgemeinuntersuchung erhobenen Gesamtzustand des Patienten.

\section{Therapie der akuten Pankreatitis}

\section{Flüssigkeitstherapie}

Vomitus und Inappetenz führen schnell zu einem Flüssigkeitsdefizit, das mittels einer an Dehydratationsgrad ( $\triangleright$ Tab. 2) und Flüssigkeitsverlust angepassten Infusionstherapie (Kasten) ausgeglichen werden muss. Während die Dehydratation auf zellulärer Ebene eher langsam (innerhalb von 24 Stunden) ausgeglichen wird, sollte eine in der Regel schnell entstandene Hypovolämie mittels einzelner Boli (10-20 ml/kg pro über 15 min) bis zur Normalisierung der Vitalparameter zügig ausgeglichen werden.

Dazu sind in erster Linie kristalloide Vollelektrolytlösungen, z. B. Sterofundin ${ }^{\circledR}$, Deltajonin ${ }^{\circledR}$, Ringer-Laktat, geeignet. In einer humanmedizinischen Studie zeigte die Verwendung einer leicht alkalisierenden Lösung wie Ringer-Laktat einen positiven Effekt, da durch den $\mathrm{pH}$ Anstieg eine weitere Trypsin-Aktivierung in den Zellen des Pankreas vermindert wird [27]. Beim Hund gibt es diesbezüglich keine Studien.

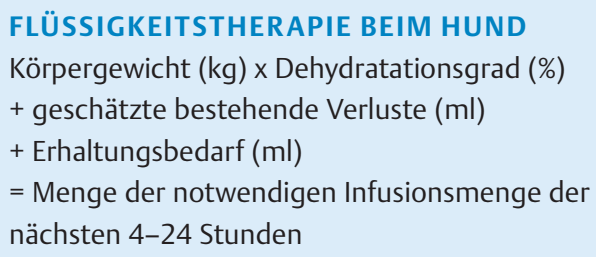

Beispiel: Hund $15 \mathrm{~kg}, 10 \%$ Dehydratationsgrad, ca. $500 \mathrm{ml}$ Verluste (z. B. durch Erbrechen)

1. Dehydrationsausgleich: $15 \times 10 \% \times 10=1500 \mathrm{ml}$

2. Verluste: $+500 \mathrm{ml}$

3. Erhaltungsbedarf*: $(15 \mathrm{~kg} \times 30)+70=520 \mathrm{ml} / \mathrm{Tag}$

$=2520 \mathrm{ml} / 24$ Stunden $(105 \mathrm{ml} / \mathrm{h})$

* Berechnung des täglichen Erhaltungsbedarfs bei Tieren unter $2 \mathrm{~kg}$ und über $50 \mathrm{~kg}$ : (Körpergewicht $\left.[\mathrm{kg}]^{0,75}\right) \times 70$ [32]

\section{Analgetische Therapie}

Eine akute Pankreatitis ist meist eine sehr schmerzhafte Erkrankung, auch wenn einige Hunde keine typischen Schmerzanzeichen zeigen. Zu empfehlende Analgetika sind in erster Linie Opioide wie Butorphanol, Buprenorphin, Fentanyl oder Tramadol ( $\bullet$ Tab. 3) $[8,10,27]$. Nicht steroidale Antiphlogistika sollten bei einer akuten Pan- 
- Tab. 2 Ermittlung des Dehydratationsgrads [32].

\begin{tabular}{|l|l|}
\hline $\begin{array}{l}\text { Dehydrata- } \\
\text { tionsgrad (\%) }\end{array}$ & klinische Symptome \\
\hline$<5$ & keine klinischen Auffälligkeiten \\
\hline $5-8$ & - trockene Schleimhäute \\
& - verminderter Hautturgor \\
\hline $8-10$ & - verminderter Hautturgor \\
& - trockene Schleimhäute \\
\hline & - ggr. verlängerte kapilläre Füllungszeit \\
\hline $10-12$ & - Hautfalte bleibt stehen \\
\hline & - trockene Schleimhäute \\
\hline & - verzögerte kapilläre Füllungszeit \\
\hline & - Angen eingesunken \\
\hline 12 & - alles oben Genannte \\
& - Schock oft lebensbedrohlich \\
\hline
\end{tabular}

kreatitis aufgrund der bestehenden Dehydratation und Hypovolämie nicht eingesetzt werden.

\section{Gabe von Fresh Frozen Plasma (FFP)}

FFP enthält viele Gerinnungsfaktoren, antiinflammatorische Substanzen sowie Albumin. Die Gabe von FFP erscheint somit bei einer fulminanten Pankreatitis, insbesondere in Zusammenhang mit einer schweren Hypoalbuminämie und DIC logisch. Jedoch gibt es bisher keine ausreichenden Studien, die belegen, dass die Gabe von FFP $(10-15 \mathrm{ml} / \mathrm{kg}$ i.v.) bei Hunden mit Pankreatitis von Vorteil ist $[10,27]$. In der Humanmedizin wird FFP nach aktuellen Guidelines zur Transfusion von FFP nur bei Pa- tienten mit aktiven Blutungen oder vor einer Operation bei Patienten mit einer Koagulopathie eingesetzt. In der Veterinärmedizin fehlen klare evidenzbasierte Richtlinien zum Einsatz von FFP. Der Einsatz sollte für jeden Patienten individuell abgewogen werden [36]. Ein Einsatz im Rahmen einer Pankreatitis ist nicht empfehlenswert.

\section{Antiemetische Therapie}

Die antiemetische Therapie ( $\bullet$ Tab. 3 ) ist ein wichtiger Bestandteil der Therapie der Pankreatitis, um den Flüssigkeitshaushalt und die Elektrolyte zu stabilisieren sowie eine gute Futteraufnahme zu gewährleisten.

Maropitant ist ein Neurokinin-1-Rezeptorblocker, der die Produktion der Substanz P blockiert. Letztere spielt eine wichtige Rolle in der Schmerzentwicklung. Bei einer Entzündung des Pankreas kommt es zu einer vermehrten Sekretion von Substanz $P$ und einer Hochregulation der Neurokinin-1-Rezeptoren [29]. Damit ist Maropitant ein sehr effektives Antiemetikum in der Therapie der akuten Pankreatitis und zeigt zudem einen analgetischen Effekt bei viszeralem Schmerz.

Metoclopramid (MCP) ist kein starkes Antiemetikum und reicht in der Regel bei einer Pankreatitis nicht aus. Als Dauertropfinfusion zeigt MCP eine bessere antiemetische Wirksamkeit als bei Bolusgabe sowie einen prokinetischen Effekt.

\section{Antimikrobielle Therapie}

Eine primäre bakterielle Ursache für die akute Pankreatitis beim Hund ist sehr selten. Es gibt zudem bisher keinen Hinweis darauf, dass die prophylaktische Gabe von Anti-

- Tab. 3 Dosierungsempfehlungen beim Hund.

\begin{tabular}{|c|c|c|}
\hline Wirkstoff & Handelsname & Dosierung \\
\hline \multicolumn{3}{|l|}{ Antibiotikum } \\
\hline Amoxicillin/Clavulansäure & Clavaseptin $^{\circledR}$ & $12,5-25 \mathrm{mg} / \mathrm{kg} 2 \times \mathrm{tgl}$. i.v./p.o. \\
\hline Marbofloxacin & Marbocyl ${ }^{\circledR}$ & 2,75-5,55 mg/kg $1 \times$ tgl. i.v./p.o. \\
\hline \multicolumn{3}{|l|}{ Antiemetika } \\
\hline Maropitant & Cerenia $^{\circledR}$ & $1 \mathrm{mg} / \mathrm{kg} 1 \mathrm{x}$ tgl. i.v./s.c. oder $2 \mathrm{mg} / \mathrm{kg} 1 \times$ tgl. p. o. \\
\hline Metoclopramid & Emeprid $^{\circledR}$ & 0,2-0,5 mg/kg i.v./i. m./p. o. 3-4x tgl., als DTI 0,3 mg/kg/h i.v. \\
\hline Ondansetron & Zofran $^{\circledR}$, Cellondan ${ }^{\circledR}$ & $0,5-1,0 \mathrm{mg} / \mathrm{kg} 2 \times \mathrm{tgl}$. i.v. \\
\hline \multicolumn{3}{|l|}{ Opiode } \\
\hline Buprenorphin & Temgesic $^{\circledR}$, Bupresol ${ }^{\circledR}$ & $0,006-0,02 \mathrm{mg} / \mathrm{kg} 3-6 \times \mathrm{tg}$ l. s.c./i.v. \\
\hline Butorphanol & Dolorex $^{\circledR}$, Morphasol $^{\circledR}$, Torbugesic ${ }^{\circledR}$ & $0,2-0,4 \mathrm{mg} / \mathrm{kg}$ alle $2-4$ Stunden i.v./i. m./s. c. \\
\hline Fentanyl & Fentany $\left.\right|^{\circledR}$ & $0,01 \mathrm{mg} / \mathrm{kg}$ i.v./i. m./s.c. alle 2 Stunden, als DTI $2-6 \mu \mathrm{g} / \mathrm{kg} / \mathrm{h} \mathrm{i.v.}$ \\
\hline Tramadol & Tramado $^{\circledR}$ & 5 mg/kg 3-4 x tgl. p.o. \\
\hline \multicolumn{3}{|l|}{ Protonenpumpenblocker } \\
\hline Omeprazol & Omeprazol $^{\circledR}$ & 0,7 mg/kg 1-2 x tgl. i.v./p.o. \\
\hline Pantoprazol & Pantoprazo ${ }^{\circledR}$ & 0,5 mg/kg 1-2 x tgl. i.v./p.o. \\
\hline
\end{tabular}


biotika einen positiven Einfluss auf die Genesung hat. Der Routineeinsatz von Antibiotika ist daher nicht zu empfehlen.

Bei einer fulminanten akuten Pankreatitis kann es jedoch zu einer bakteriellen Translokation aus dem Darm kommen [37,38]. Bei Hinweisen auf ein septisches Geschehen ist eine antimikrobielle Therapie angezeigt. Dafür eignet sich z.B. intravenös Amoxicillin/Clavulansäure $(\vee$ Tab. 3) [27].

\section{Protonenpumpenblocker}

Es gibt aktuell keine Studie, die belegt, dass eine Reduktion der Magensäure den Verlauf einer Pankreatitis positiv beeinflusst. Bei Hinweisen auf eine Ösophagitis oder Magenulzerationen ist eine Therapie mit Protonenpumpenblockern wie Omeprazol und Pantoprazol ( $>$ Tab.3) indiziert. Orale Dosen von Omeprazol alle 12 Stunden zeigten, verglichen mit Pantoprazol und Ranitidin, den stärksten Anstieg des pH-Werts, der auch am längsten anhielt $[27,28]$.

\section{Ernährung}

Das Dogma der Nahrungskarenz bei einer akuten Pankreatitis ist heute nicht mehr haltbar.

\section{Merke}

Der frühzeitige Beginn einer kontrollierten Fütterung hat einen positiven Effekt auf den Verlauf der Erkrankung.

Hierzu sollte nur eine streng fettarme Diät, z.B. Hills ${ }^{\circledR}$ Prescription diet i/d low fat, Royal Canin ${ }^{\mathrm{TM}}$ Gastro Intestinal low fat, eingesetzt werden (<3 g Fett/100 kcal [31]).

Bei ausbleibender Futteraufnahme ist die Fütterung über Nasen-Schlund-Sonde, Ösophagus- oder Magensonde angezeigt. Eine partielle oder totale parenterale Ernährung kann notwendig werden. Die enterale Fütterung sollte jedoch der parenteralen Ernährung bevorzugt werden, da sich die klinische Symptomatik, vor allem das Erbrechen, mit einer frühzeitigen Futteraufnahme schneller verbessert $[8,10,27,30]$. Als unterstützende symptomatische Therapie zur Steigerung des Appetits kann die Gabe von Mirtazapin, einem tetrazyklischen Antidepressivum, oder Cyproheptadin, einem SerotoninAntagonisten, hilfreich sein.

Eine Therapie mit Pankreasenzymen oder Glukokortikoiden ist bei einer akuten Pankreatitis nicht indiziert.

\section{Therapie der chronischen Pankreatitis}

Die Therapie der chronischen Pankreatitis besteht ebenfalls vor allem in der Behandlung zugrunde liegender Erkrankungen und dem Abstellen möglicher Risikofaktoren. Je nach Schweregrad der gezeigten klinischen Symptome ist eine unterstützende Therapie wie bei der akuten Pankreatitis indiziert. Auch bei einer chronischen Pankreatitis sollte eine fettarme Diät gefüttert werden.

\section{PRAXISTIPP}

Bei Patienten, bei denen aufgrund von anderen Grunderkrankungen eine spezielle Diät notwendig ist, ist es sinnvoll, einen Spezialisten für Tierernährung zu konsultieren, um eine individuelle Diät zu erstellen.

\section{Fazit}

Für die sichere Diagnose einer akuten Pankreatitis beim Hund ist meist eine Kombination aus klinischer Untersuchung, Bestimmung der DGGR-Lipase/cPLI/Spec cPL sowie abdominaler Sonografie notwendig. Die Diagnose der chronischen Pankreatitis ist schwierig und erfordert meist eine Biopsie. Die Therapie richtet sich in beiden Fällen vor allem nach der klinischen Symptomatik und nach den zugrunde liegenden Erkrankungen. Bei Tieren mit einer schweren, akuten Pankreatitis ist eine angepasste Infusionstherapie sehr wichtig. Die Fütterung einer streng fettarmen Diät nimmt sowohl bei der akuten als auch bei der chronischen Pankreatitis einen hohen Stellenwert ein.

\section{Korrespondenzadresse}

\author{
Dr. Diana Dorn \\ Dr. Susanne Mangelsdorf, Dipl. ECVIM-CA \\ Fachtierärztin für Innere Medizin der Kleintiere \\ Kleintierspezialisten Überweisungszentrum \\ Dres. Schmerbach \& Höpfner GmbH \\ Wittestraße 30 Haus $P$ \\ 13509 Berlin \\ kontakt@kleintierspezialisten.de
}

Literatur

[1] Bradley EL. A clinically based classification system for acute pancreatitis. Arch Surg 1993; 128: 586-590

[2] Newman SJ, Steiner JM, Woosley K et al. Histologic assessment and grading of the exocrine pancreas in the dog. J Vet Diagn Invest 2006; 18: 115-118,[3]

Spillmann T. Pancreatitis: Etiology and pathophysiology. In: Ettinger SJ, Feldman EC, Coté E, eds. Textbook of Veterinary Internal Medicine. St. Louis: Elsevier; 2017: 1681-1682

[4] Mansfield C. Acute pancreatitis in dogs: advances in understanding, diagnostics, and treatment. Top Companion Anim Med 2012; 27: 123-132

[5] Watson PJ, Roulois AJ, Scase T et al. Characterization of chronic pancreatitis in English Cocker Spaniels. J Vet Intern Med 2011; 25: 797-804

[6] Watson P. Pancreatitis in dogs and cats: definitions and pathophysiology: review. J Small Anim Pract 2015; 56: 3-12

[7] Xenoulis PG. Diagnosis of pancreatitis in dogs and cats. J Small Anim Pract 2015; 56: 13-26 
[8] Steiner JM. Exocrine pancreas. In: Steiner JM, ed. Small Animal Gastroenterology. Hannover: Schlütersche; 2008: 283-294

[9] Hess RS, Saunders HM, Van Winkle TJ et al. Clinical, clinicopathologic, radiographic, and ultrasonographic abnormalities in dogs with fatal acute pancreatitis: 70 cases (1986-1995). J Am Vet Med Assoc 1998; 213: 665-670

[10] Steiner JM. Canine Pancreatitis: Diagnosis and treatment. In: Ettinger SJ, Feldman EC, Coté E, eds. Textbook of Veterinary Internal Medicine. St. Louis: Elsevier; 2017: 1681-1682

[11] Willard MD, Tvedten H. Small Animal Clinical Diagnosis by Laboratory Methods. $5^{\text {th }}$ ed. St. Louis: Elsevier; 2012

[12] Graca R, Messick J, McCullough $S$ et al. Validation and diagnostic efficacy of a lipase assay using substrate 1,2-o-dilaurylrac-glycero glutaric acid-(6'methyl resorufin)-ester for the diagnostic of acute pancreatitis in dogs. Vet Clin Path 2005; 34: $39-43$

[13] Trivedi S, Marks SL, Krass PH et al. Sensitivity and specificity of canine pancreas-specific lipase (cPL) and other markers for pancreatitis in 70 dogs with and without histopathologic evidence of pancreatitis. J Vet Intern Med 2011; 25: 1241-1247

[14] Yuki M, Hirano T, Nagata $N$ et al. Clinical utility of diagnostic laboratory tests in dogs with acute pancreatitis: a retrospective investigation in a primary care hospital. J Vet Intern Med 2016; 30: 116-122

[15] Larson MM. Ultrasound imaging of the hepatobiliary system and pancreas. Vet Clin North Small Anim Pract 2016; 46: 453-480

[16] Steiner JM, Newman S, Xenoulis P et al. Sensitivity of serum markers for pancreatitis in dogs with macroskopic evidence of pancreatitis. Vet Ther 2008; 9: 263-272

[17] Kim DH, Pickhardt PJ. Radiologic assessment of acute and chronic pancreatitis. Surg Clin North Am 2007; 87: 13411358

[18] Scaglione M, Casciani E, Pinto A et al. Imaging assessment of acute pancreatitis: a review. Semin Ultrasound CT MR 2008; 29: $322-340$

[19] Fossard JR, Steer ML, Pastor CM. Acute pancreatitis. Lancet 2008; 371: 143-152

[20] Cappel MS. Acute pancreatitis: etiology, clinical presentation, diagnosis, and therapy. Med Clin North Am 2008; 92: 889923

[21] Wahab SS, Khan RA, Ahmad I et al. Imaging and clinical prognosis indicators of acute pancreatitis a comparative insight. Acta Gastroenterol Latinoam 2010; 40: 283-287

[22] Adrian AM, Twedt DC, Kraft SL et al. Computed tomographic angiography under sedation in diagnosis of suspected canine pancreatitis: a pilot study. J Vet Intern Med 2015; 29: 97-103

[23] Cordner AP, Armstrong PJ, Newman SJ et al. Effect of pancreatic tissue sampling on serum pancreatic enzyme levels in clinically healthy dogs. J Vet Diagn Invest 2010; 22: 702-707

[24] Cowell RL, Valenciano AC. Cowell and Tyler's Diagnostic Cytology and Hematology of the Dog and Cat. St. Louis: Elsevier; 2014
[25] Lidbury JA, Suchodolski JS. New advances in the diagnosis of canine and feline liver and pancreatic disease. Vet J 2016; 215: 87-95

[26] Xenoulis PG, Steiner JM. SNAP tests for pancreatitis in dogs and cats: SNAP canine pancreatic lipase and SNAP feline pancreatic lipase: review. Top Companion Anim Med 2016; 31: 134-139

[27] Mansfield C, Beths T. Management of acute pancreatitis in dogs: critical appraisal with focus on feeding and analgesia: review. J Small Anim Pract 2015; 56: 27-39

[28] Bersenas AM, Mathews KA, Allen DG et al. Effects of ranitidine, famotidine, pantoprazole, and omeprazole on intragastric $\mathrm{pH}$ in dogs. Am J Vet Res 2005; 66 (3): 425-431

[29] Koh YH, Bhatia M. Sustance P (SP). Im Internet: https://pancreapedia.org/sites/default/files/substance_P_pdf_4-16-14. pdf; Stand: Dezember 2017

[30] Mansfield CS, James FE, Steiner JM et al. A pilot study to assess tolerability of early enteral nutrition via esophagostomy tube feeding in dogs with severe acute pancreatitis. J Vet Intern Med 2011; 25: 419-425

[31] Jensen KB, Chan DL. Nutritional management of acute pancreatitis in dogs and cats. J Vet Emerg Crit Care 2014; 24: 240-250

[32] Silverstein D. Daily Intravenous fluid therapy. In: Silverstein D, Hopper K, eds. Small Animal Critical Care Medicine. St. Louis: Elsevier; 2009: 272-273

[33] Graca R, Messick ], McCullough S et al. Validation and diagnostic efficacy of a lipase assay using the substrate1,2-o-dilaurylrac-glycero glutaric acid-(6'-methylresorufin)-ester for the diagnosis of acute pancreatitis in dogs. Vet Clin Pathol 2005; 34: $39-43$

[34] Kook PH, Kohler N, Hartnack S et al. Agreement of serum spec CPL with the 1,2-o-dilauryl-rac-glycero glutaric acid-(6'-methylresorufin) ester (DGGR) lipase assay and with pancreatic ultrasonography in dogs with suspected pancreatitis. J Vet Intern Med 2014; 28: 863-870

[35] Mansfield AS, Watson PD, Jones BR. Specificity and sensitivity of serum canine pancreatic elastase- 1 concentration in the diagnosis of pancreatitis. J Vet Diagn Invest 2011; 23(4): 691-697

[36] Beer KS, Silverstein DC. Controversies in the use of fresh frozen plasma in critically ill small animal patients. J Vet Emerg Crit Care 2015; 25 (1): 101-106

[37] Liu Q, Djuricin G, Nathan C et al. The effect of interleukin-6 on bacterial translocation in acute canine pancreatitis. Int J Pancreatol 2000; 27 (2): 157-165

[38] Balzan S, De Almeida Quadros C, De Cleva R et al. Bacterial translocation: Overview of mechanisms and clinical impact. Review J Gastroenterol Hepatol 2007; 22: 464-471

Bibliografie

DOI https://doi.org/10.1055/s-0043-124118

kleintier konkret 2018; 21: 20-31 @ Georg Thieme Verlag KG Stuttgart · New York ISSN 1434-9132 


\section{Fragebogen}

\section{Frage 1}

Bei welcher Hunderasse wird die Pankreatitis als Folge einer chronischen immunvermittelten Entzündung diskutiert?
A Zwergschnauzer
B Zwergpudel
C Englischer Cocker Spaniel
D Deutscher Schäferhund
E Riesenschnauzer

\section{Frage 2}

Welche therapeutische Maßnahme ist bei einer akuten Pankreatitis immer indiziert?
A antimikrobielle Therapie
B Kortikosteroide
C Nahrungskarenz
D totale parenterale Ernährung (TPN)
E Flüssigkeitstherapie mit Vollelektrolytlösungen

\section{Frage 3}

Welches ist kein begünstigender Risikofaktor für die Entstehung einer Pankreatitis?
A Hyperadrenokortizismus
B Phenobarbital
C Adipositas
D Hypokaliämie
E Hypertriglyzeridämie

\section{Frage 4}

Welches Testverfahren ist für die Diagnose der Pankreatitis besonders geeignet?
A TLI und Sonografie
B cPLI oder DGGR-Lipase
C Serum-Amylase-Aktivität und Röntgen
D cPL-Snap-Test und Röntgen
E Blutchemie und Sonografie

\section{Frage 5}

Mit welchem Verfahren wird die chronische Pankreatitis sicher diagnostiziert?
A Sonografie der Pankreas
B Röntgen des Abdomens
C Computertomografie
D histopathologische Untersuchung einer Pankreasbiopsie
E zytologische Untersuchung eines Pankreas-Feinnadelaspirats

\section{Frage 6}

Was ist die häufigste Ursache einer Bilirubinämie im Verlauf einer Pankreatitis?
A extravasale Hämolyse
B intrahepatische Gallengangsobstruktion
C extrahepatische Gallengangsobstruktion
D Leberversagen
E Cholezystitis

\section{Frage 7}

Was zeigt ein positiver CPL-Snap-Test an?
A Dass eine akute Pankreatitis ausgeschlossen werden kann.
B Dass eine akute Pankreatitis sicher vorliegt.
C Dass eine chronische Pankreatitis sicher vorliegt.
D Dass die cPL über einem Wert von $200 \mu \mathrm{g} /$ l liegt.
E Dass eine chronische Pankreatitis ausgeschlossen werden kann.

\section{Frage 8}

Was ist bei der Fütterung von Pankreatitispatienten zu beachten?

A Nahrungskarenz über einige Tage, dann Anfüttern mit einer fettreduzierten Kost.

B Legen einer Ernährungssonde und Gabe einer hochkalorischen fettreichen Kost, um Gewichtsverlusten vorzubeugen.

C Es sollte bei schwer kranken Patienten immer die totale parenterale Ernährung (TPN) vorgezogen werden.

D Die Fütterung spielt keine Rolle, es kann alles gefüttert werden, Hauptsache der Hund frisst.

E Die Futteraufnahme ist sehr wichtig, es sollte unbedingt auf eine fettarme Diät geachtet werden.

\section{Frage 9}

Welches ist kein typisches Symptom einer akuten Pankreatitis?
A Anorexie
B PU/PD
C Vomitus
D Gewichtsverlust
E Diarrhoe

\section{Frage 10}

Welche analgetische Therapie ist bei Patienten mit Pankreatitis sinnvoll?
A Meloxicam
B Carprofen
C Phenylbutazon
D Buprenorphin
E Pankreatitispatienten brauchen nur in Ausnahmefällen eine analgetische Therapie 


\section{Pankreatitis beim Hund}

Diana Dorn, Susanne Mangelsdorf

\section{A Lernerfolgskontrolle}

\section{Bitte kreuzen Sie die richtigen \\ Antworten an! Es ist jeweils nur 1 Antwort pro Frage richtig!}

\begin{tabular}{l|l|l|l|l|l|l|} 
Frage 1 & a & b & c & d & e \\
\hline Frage 2 & a & b & c & d & e \\
\hline Frage 3 & a & b & c & d & e \\
\hline Frage 4 & a & b & c & d & e \\
\hline Frage 5 & a & b & c & d & e \\
\hline
\end{tabular}

\begin{tabular}{l|l|l|l|l|l|l|} 
Frage 6 & a & b & c & d & e \\
\hline Frage 7 & a & b & c & d & e \\
\hline Frage 8 & a & b & c & d & e \\
\hline Frage 9 & a & b & c & d & e \\
\hline Frage 10 & a & b & c & d & e \\
\hline
\end{tabular}

\section{B Teilnehmer}

Titel | Name | Vorname
Straße | Hausnummer

Beruf

\section{ATF

